\title{
Simultaneous cell cycle and phenotypic analysis of primary uveal melanoma by flow cytometry
}

J. LAWRY, M.O. SMITH, A.J. PARSONS, I.G. RENNIE

\begin{abstract}
Background and purpose DNA ploidy and cell
\end{abstract} cycle measurements of uveal melanoma tissue are regarded as having limited prognostic significance. In contrast, dual-parameter (DNA monoclonal antibody) flow cytometry offers a convenient and rapid way to screen tumour samples for a variety of phenotypic markers, whilst simultaneously measuring DNA ploidy and cell cycle, and therefore has the increased potential to identify clinically relevant indicators of disease progression. The aim of the present study was to identify a simple yet robust method for isolating, preserving and staining cells that could be analysed by flow cytometry.

Methods Using a simple preparation procedure, a panel of membrane-associated antibodies (ICAM-1, W632, HLA-DR) and nuclear or cytoplasmic oncoprotein antibodies (c-erbB-2, c-myc, bcl-2, p53), together with positive (PHM-5) and negative (FITC $F\left(a^{\prime}\right)_{2}$ ) controls, were assayed. It was considered important to test the protocol with markers expressed on the cell surface, and in the cytoplasm and nucleus, so as not to be restrictive and thereby exclude an antigen of potential clinical interest. In addition, such panels would also enable the generation of a 'phenotypic profile' for each specimen that may reveal clinically significant trends.

Results Our results indicate that tissue dissociation followed by brief fixation in $1 \%$ paraformaldehyde and permeabilisation in $\mathbf{7 0} \%$ methanol produces a stable single cell suspension, which can subsequently be stained with a wide range of antibodies for the accurate identification of cells in a potentially heterogeneous tumour population.

Conclusion This technology can rapidly identify sub-populations of cells expressing differing levels of proteins, which may prove to be indicative of disease progression for this aggressive disease.
Key words DNA ploidy, Flow cytometry, Melanoma, Monoclonal antibody

Uveal melanoma is reported to be the most common primary ocular neoplasm in adults. The 15-year survival rate for patients treated by enucleation is approximately $50 \%,{ }^{1}$ and this cancer preferentially metastasises to the liver.

Neoplastic progression is dependent on the control, or loss of control, of growth. This may be highly relevant to malignant progression and the development of metastases, which in the case of ocular melanoma preferentially occur in the liver. Immunohistochemical studies of fresh uveal melanoma samples are limited, but have included the analysis of bromodeoxyuridine (BrdU) labelling, and the measurement of DNA ploidy and cell cycle. The published findings suggest that BrdU may be of value in identifying proliferating cells following unsuccessful irradiation treatment, ${ }^{2}$ whilst studies on proliferation indicate that cell turnover can not be correlated with tumour size or pathology. ${ }^{3,4}$ Studies on fresh samples of metastatic cutaneous malignant melanoma suggest that tumour ploidy and S-phase fraction are independent prognostic variables, and major determinants of survival after first recurrence, ${ }^{5}$ whilst patients with aneuploid tumours and low S-phase fractions had improved survival following systemic therapy suggesting that these tumour types were more sensitive to therapy. In addition, a sequential analysis of metastatic cutaneous lesions indicated that, with time, the evolution of an aneuploid genotype was associated with reduced survival. ${ }^{6}$

Thus far, the use of flow cytometry for the analysis of DNA ploidy and cell cycle in uveal melanoma has largely been restricted to the extraction and analysis of nuclei from sections of paraffin-embedded tissue. ${ }^{7}$ Whilst these measurements may provide basic information on the DNA content and proliferation of tumour populations, evaluation of such data can be
J. Lawry

M.O. Smith

Institute for Cancer Studies University Medical School Beech Hill Road Sheffield S10 2RX, UK Tel: +44 (0)1142713023 Fax: +44 (0)1142713515 e-mail: j.lawry@sheffield.ac.uk

A.J. Parsons

I.G. Rennie

Department of Ophthalmology

Royal Hallamshire Hospital Sheffield S10 2JF, UK

The flow cytometry unit in the University Medical School in Sheffield is generously funded by the Yorkshire Cancer Research Campaign 
difficult without appropriate diploid controls, and the technique is still restricted to single-parameter analysis, making it impossible to further characterise subpopulations of cells, or indeed to quantify the actual tumour versus stromal/infiltrative component of the sample.

In an attempt to increase the clinical impact of flow cytometric DNA analysis, antibody staining was added to enable the simultaneous bivariate analysis of DNA ploidy and antigen expression together with the degree of antibody binding in each measurable phase of the cell cycle. ${ }^{8}$ This technique has proved to be valuable in the identification of rare aneuploid populations using surface antigens in haematological malignancies; ${ }^{9}$ and by using cytokeratin antibodies for epithelial tumours. ${ }^{10,11}$ The technique has not been widely applied to uveal melanoma, nor has the application of panels of antibodies against membrane, cytoplasmic and nuclear antigens been evaluated using a single protocol.

The purpose of the present study was therefore to identify whether it was possible to take fresh tissue specimens and dissociate the cells sufficiently to produce a single cell suspension. Secondly, it was considered important to see whether cells could then be stained with specific monoclonal antibodies, simultaneously with a DNA fluorochrome, in order to subdivide heterogeneous populations of cells into several categories: (i) antibody positive or negative, (ii) DNA diploid or aneuploid, (iii) cell cycling or non-cycling populations, (iv) antibodystained diploid or aneuploid cells, and (v) antibodystained cycling or non-cycling cells.

The findings of the present study suggest that a detailed 'tumour profile' can be determined from dissociated specimens of fresh or frozen uveal melanoma, but the range of antibodies used in the panel is dependent upon the size of the tumour and consequently the tumour cell yield. We present illustrative examples of the nature of antibody/DNA staining on only four cell suspensions taken from individual tumour samples, to prove the validity of the preparation technique. We are therefore not able to make clinical judgement on the results nor even to determine the best antibodies to use in the clinical analysis of these tumours. Further studies are now being undertaken on a larger group of tumour samples to question whether there are any possible relationships between traditional clinical and pathological parameters (tumour cell type, volume, location (posterior/anterior to the equator), the tissue origin (choroid, ciliary body), and the flow cytometric measurement of cell surface protein or cytoplasmic/nuclear oncoprotein expression in cells taken from samples of primary uveal melanoma.

\section{Materials and methods}

\section{Patient group}

Fresh tumour tissue was collected following enucleation, transported in liquid nitrogen to the laboratory, and then either stored or dissociated to produce a single-cell suspension. Patient details and clinical information were recorded in a data base. Pathological analysis was undertaken by an experienced ophthalmic pathologist (M.A.P.), and results recorded.

\section{Tissue preparation}

Samples of primary ocular melanoma collected following enucleation were divided for storage, histology or immediate use. Single-cell suspensions were generated by dicing pieces of fresh tissue with scalpels in phosphate-buffered saline (PBS) containing antibiotics. Cells for flow cytometric analysis were washed in PBS, fixed in $1 \%$ paraformaldehyde at room temperature for 5 min with frequent mixing, washed in PBS and permeabilised in $70 \%$ methanol cooled to $4{ }^{\circ} \mathrm{C}$, for a further $15 \mathrm{~min}$ period at room temperature. Cells were then either stored at $4{ }^{\circ} \mathrm{C}$ or washed and stained immediately.

\section{Flow cytometric staining}

Cell suspensions were washed in PBS then PBS containing $1 \%$ newborn calf serum (PBS/serum), $10^{6}$ cells aliquoted into tubes, and the primary (test) antibody added at an appropriate concentration (typically 5 or $10 \mu \mathrm{l}$ neat antibody). Staining was carried out for $30 \mathrm{~min}$ at $4{ }^{\circ} \mathrm{C}$, followed by washing in PBS/ serum. The secondstage fluorescein isothiocyanate-conjugated goat antimouse antibody (FITC-F(ab') ${ }_{2}$, Caltag, Bradsure Biologicals, Loughborough, UK) was then added (100 $\mu$ l at $1 / 100$ dilution in PBS/serum) for a further $30 \mathrm{~min}$ at $4{ }^{\circ} \mathrm{C}$ (protected from light).

The samples were then washed in PBS/serum to remove excess antibody, and the pellet resuspended. The DNA fluorochrome, propidium iodide (PI, Sigma, Dorset, UK), was added (300 $\mu \mathrm{l}$ at $50 \mu \mathrm{g} / \mathrm{ml}$ in PBS) for at least $30 \mathrm{~min}$ (protected from light), together with $50 \mu \mathrm{l}$ RNAse ( $5 \mathrm{u} / \mathrm{ml}$, Sigma, Dorset, UK), after which samples were syringed (25G needle) and filtered through a $50 \mu \mathrm{m}$ stainless steel mesh to remove any large aggregates of cells before analysis on the flow cytometer. In most cases the quality of DNA staining (peak CV) was improved by overnight storage at $4{ }^{\circ} \mathrm{C}$ in the final buffer containing PI DNA dye.

\section{Monoclonal antibodies assessed}

The following panel of antibodies were used to determine the feasibility of the technique, with care taken to include antibodies against cell surface, cytoplasmic and nuclear antigens:

Negative control: FITC second-stage goat anti-mouse $\mathrm{F}\left(\mathrm{ab}^{\prime}\right)_{2}$ antibody (Caltag, Bradsure Biologicals, Loughborough, UK).

Positive control: PHM-5 epithelial/endothelial antibody (Silenus, Bio-Stat Diagnostics, Stockport, UK).

W632 MHC Class I antibody (a generous gift from the BTS labs., Sheffield). 
HLA-DR MHC Class II antibody CR3/43 (DAKO, High Wycombe, UK).

ICAM-1 (CD54) adhesion molecule antibody (a generous gift from ICRF, London).

c-erb-B2 cytoplasmic domain antibody, code: OM11952

(CRB, Cheshire, UK).

c-myc (against peptide mid-region) antibody 6E10 (CRB, Cheshire, UK).

bcl-2 antibody 124 (DAKO, High Wycombe, UK).

p53 antibody DO-7 wild-type and mutant (DAKO, High Wycombe, UK).

\section{Flow cytometry}

Samples were routinely analysed for DNA ploidy and cell cycle on an Orthocyte bench-top cytometer (Ortho Diagnostics, High Wycombe, UK). Stored files were analysed by the Multicycle DNA software analysis package on this cytometer (Phoenix Flow Systems, USA) for accurate DNA index and cell cycle calculations. Samples with diploid $G_{0} / G_{1}$ peaks having a coefficient of variation $(\mathrm{CV})$ greater than 10 were excluded from the present study, and retested if possible. The study group CV range was 3.3-9.9 (median 6.4).

Dual-stained (DNA/monoclonal antibody) samples were analysed on a FACSort bench-top cytometer (Becton Dickinson UK, Oxford, UK) by recording largeangle and forward-angle light scatter, antibody FITC fluorescence (530 nm band pass) and the DNA fluorescence of PI (620 nm long pass) for at least 10000 cells. Doublets were excluded using scatter gates, and by doublet discrimination gates using peak height and width analysis of the DNA fluorescence parameter. Measurements were made on stored data files, and included the percentage of antibody-positive cells and median level of staining, the DNA index of the sample, and cell cycle measurements. When required, DNA indices and cell cycle measurements were made using Modfit software (Verity Software House, USA).

\section{Data analysis}

Flow cytometric data were measured as median channels of fluorescence (linear fluorescence scales) and calculated mean fluorescent channels (log fluorescent scales). The FITC scales were quantified by running samples of standardised multifluorescent beads under exactly the same cytometer running conditions as used for cell samples (FluoroSpheres, DAKO, High Wycombe, UK). By calibrating fluorescence to known mean equivalent numbers of molecules of soluble fluorescein (MESF), data obtained from the cytometer on the staining intensity of antibody bound to cells can be converted into MESF values. TallyCAL software (DAKO and Applied Cytometry Systems, UK) was used to convert channel values into MESF values. By making certain assumptions (1:1 binding of the antibody to antigen; $2: 1$ binding of the secondary antibody to the primary antibody), and by recording the fluorescein:protein molar ratio of the fluoresceinated second-stage antibody (3-5 molecules
FITC:Ig molecule, goat anti-mouse $\left.\mathrm{F}\left(\mathrm{ab}^{\prime}\right) 2 \mathrm{Ig}\right)$, the calculated MESF for the stained population of cells can be converted into the approximate antigen density for each antibody used. A more accurate calculation can be made using directly conjugated monoclonal antibodies, although the fluorescence of these reagents was found to be reduced by quenching as a result of the bright PI fluorescence to a greater extent than the fluorescence of two-stage staining protocols.

\section{Results}

\section{Quantification}

Assuming 6-10 molecules FITC would bind to each primary antibody, and each primary antibody would bind to one antigen site, a median fluorescent channel of, for example, 50 would be equivalent to between 3800 and 6400 antigen sites per cell as an average for the sample population.

\section{Controls}

The first test analysed on the flow cytometer was always the negative control containing only second-stage antibody, and was used to identify the level of nonspecific staining for each sample. Clusters representing the non-stained cells were also DNA-stained using PI, so the populations identified along the $x$-axis (DNA fluorescence) were used to identify cells in $G_{1}, S$ and $G_{2} M$ phases of the cell cycle, as well as detecting the presence of aneuploidy. The lines drawn above the dot clusters in Figs. 1-3 were used to assist in the calculation of the percentage of stained cells.

The second test was the positive control used to quantitate the proportion of cells present that were of tumour origin - not infiltrating leucocytes or normal stromal cells. The latter could only be resolved if nondiploid cells were present as identified by staining with the DNA fluorochrome. PHM-5 was found to be a good positive control, generally staining over $80 \%$ of all cells in the sample. PHM-5 staining was generally bright with a median channel of 100 .

\section{Surface membrane markers}

MHC Class I (W632) staining was generally found to be restricted to a low proportion of cells in each sample (around 10\%), with a median channel in the order of 30 (data not shown). This was comparable to the staining seen with MHC Class II (HLA-DR), although the proportion of positive cells was usually higher (two of the four samples quoted in this study having $87 \%$ and $89 \%$ positive cells). Sub-populations were often seen that had different levels of staining intensity, with medians ranging from 50 to 500, or approximately 10000 to 100000 antigens per cell. One such sample is illustrated in Fig. 4.

ICAM-1 (CD54) staining was frequently seen on more than $25 \%$ cells in each sample, with differential staining apparent between different ploidy populations within the 
tumour sample. Levels of staining were generally around channel 50, as is the case for the example shown in Fig. 1 in which $65 \%$ cells are stained over background levels.

\section{Oncoprotein markers}

The fixation and permeabilisation protocol enabled the visualisation of all the internal antigens investigated in this study, with high proportions of cells staining positive for c-erb-B-2 (50-70\% cells present) with median channel measurements from 50 to 80 . c-myc (6E10) staining was evident in all samples tested at levels from $27 \%$ to $56 \%$ of cells in the four tumours illustrated (data
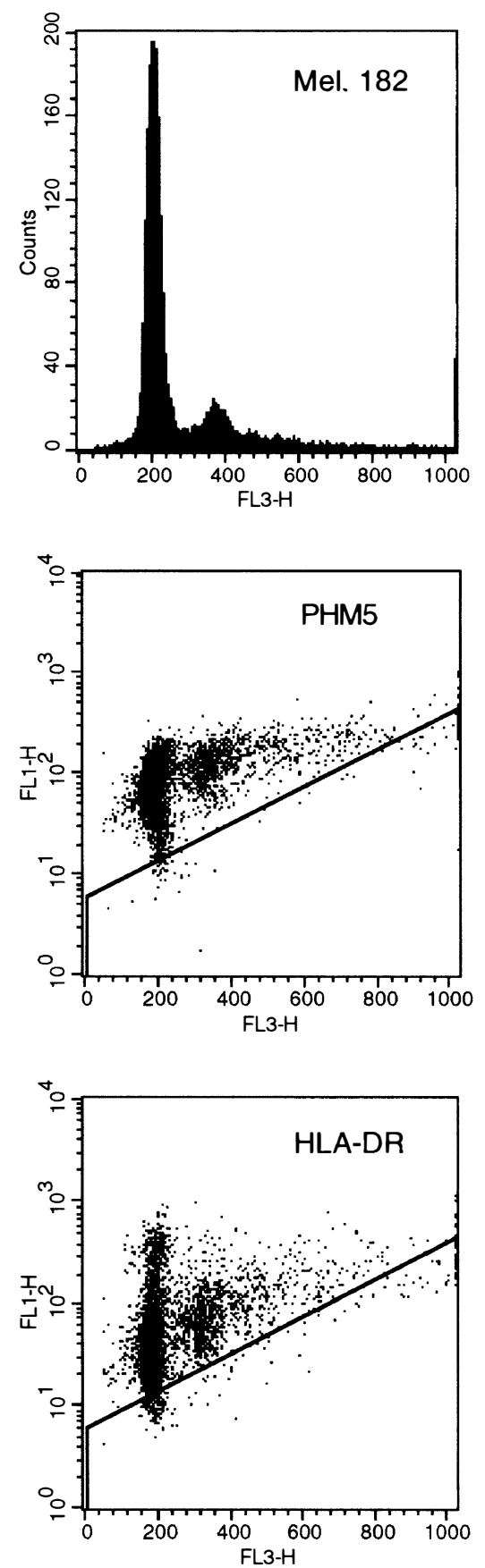

not shown), although at low intensities (channels 15-25); and $b c l-2$ staining was seen at elevated levels (i.e. $>25 \%$ ) in most samples tested, with $50 \%$ of tumours showing bcl-2 positivity on over $80 \%$ of cells present (Fig. 1). p53 staining was highly variable, the four samples cited in this study having levels of $10 \%, 27 \%, 29 \%$ and $82 \%$ p53-positive cells over background staining (Figs. 2, 3).

\section{DNA and antibody staining}

A range of samples of primary uveal melanoma were available for analysis, and Figs. 1-4 indicate the value of using dual-parameter analysis in diploid samples, where
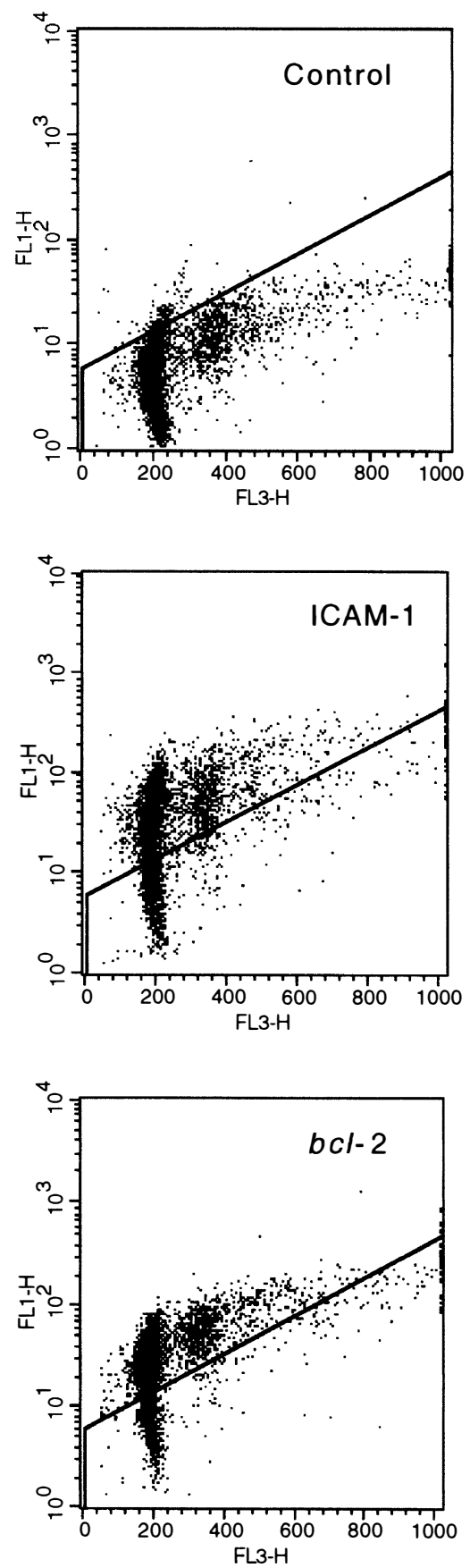

Fig. 1. The DNA profle of Mel. 182, a choroidal malignant melanoma, together with dual-parameter displays for DNA content ( $\mathrm{x}$-axis) and fluorescein isothiocyanate monoclonal antibody (FITC MoAb) staining (y-axis) for the negative control sample, the positive control (PHM-5), and the three test antibodies ICAM-1, HLA-DR and bcl-2. The diagonal line is drawn to facilitate the detection of positively stained cells: these move above the line, whilst non-staining cells remain below the line. 

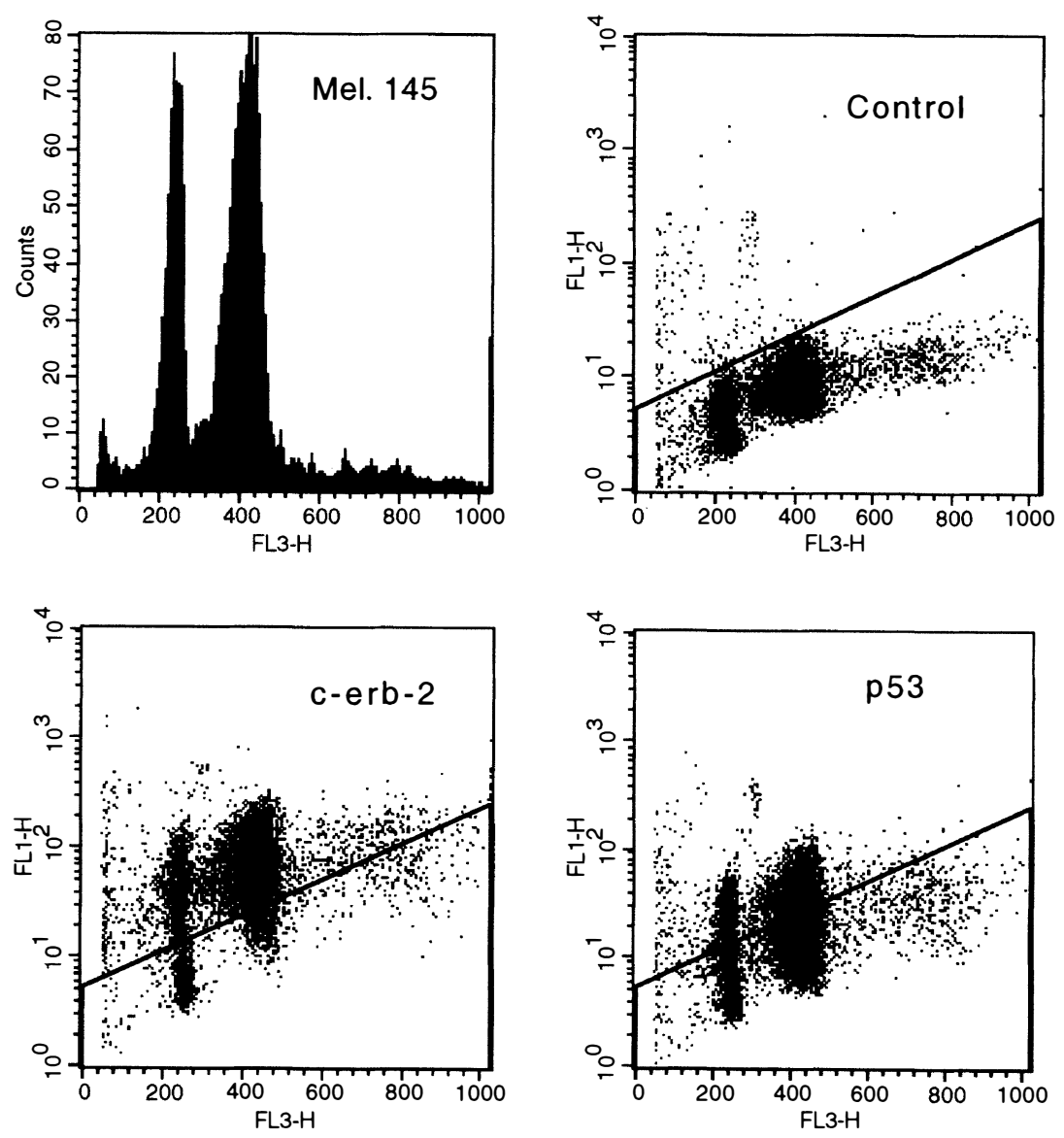

Fig. 2. Results from Mel. 145, a malignant melanoma designated as being both ciliary body and choroidal. The DNA histogram shows the tumour to contain $64 \%$ aneuploid cells with a DNA Index of 1.75. The dual-parameter (DNA/MoAb) histograms show the negative control and two oncoprotein antibodies, c-erb-B2 and p53, which stain both diploid and aneuploid cells.

it is clear that sub-populations exist with different staining patterns for the antibodies used in the test panel. In addition, in samples containing populations of cells with clear differences in DNA content, it is still possible to identify further subpopulations based on antibody staining.

Figs. 1, 2 and 3 illustrate dual-parameter staining for melanoma samples that were found to be DNA diploid (Fig. 1), diploid and aneuploid (Fig. 2) and diploid with two further aneuploid populations (Fig. 3). Fig. 1 shows the DNA profile of a choroidal malignant melanoma, together with three test antibodies and with the negative and positive control for the sample Mel.182. Whilst the majority of cells stain equally brightly with ICAM-1, HLA-DR and $b c l-2$, discrete populations from within the $\mathrm{G}_{0} / \mathrm{G}_{1}$ DNA peak clearly stain less well with ICAM-1 and $b c l-2$. In contrast, a minor population of cells from the same DNA peak stain with the HLA-DR antibody at a median channel of 300 , compared with the main population which has a median of 45 . This tumour was reported to contain $30 \%$ epitheloid cells, $70 \%$ spindle B cells. These figures correlate with the observed split in stained/ unstained cells seen with ICAM-1 and $b c l-2$ staining, with $65 \%$ and $67 \%$ staining respectively. Obviously, without cell sorting the morphology of these two staining populations can not be predicted by antibody staining alone.
Fig. 2 illustrates results from a malignant melanoma designated as being both ciliary body and choroidal, containing 64\% aneuploid cells (Mel. 145, DNA Index 1.75), where the diploid and aneuploid populations exhibit comparable levels of staining for c-erb-B2 and p53.

Fig. 3 is from an amelanotic choroidal malignant melanoma containing $75 \%$ epitheloid cells and $25 \%$ spindle B cells, and shows diploidy together with two aneuploid populations of 5.4\% (DNA Index 1.6) and $20.8 \%$ (DNA Index 2.4 ) respectively, i.e. total aneuploidy in $25 \%$ population. Whilst p53 staining was only at a low level, being close to that of the non-specific control, cells in the second aneuploid population can be seen to bind more p53 antibody than the other two populations. This tumour was highly aggressive as the patient also presented with liver metastases.

It is also technically possible to use the flow cytometer software to gate or select discrete populations of cells based on their differing DNA content, and then measure antibody binding; alternatively cells selected on the basis of differing antibody staining levels can have their DNA content and cell cycle measured. This technique is illustrated in Fig. 4 for a choroidal malignant melanoma of mixed cell pathology (10\% epitheloid, $90 \%$ spindle B cell). Data are shown in panel A as a dot display of DNA content ( $x$-axis) versus MHC Class II HLA-DR staining 

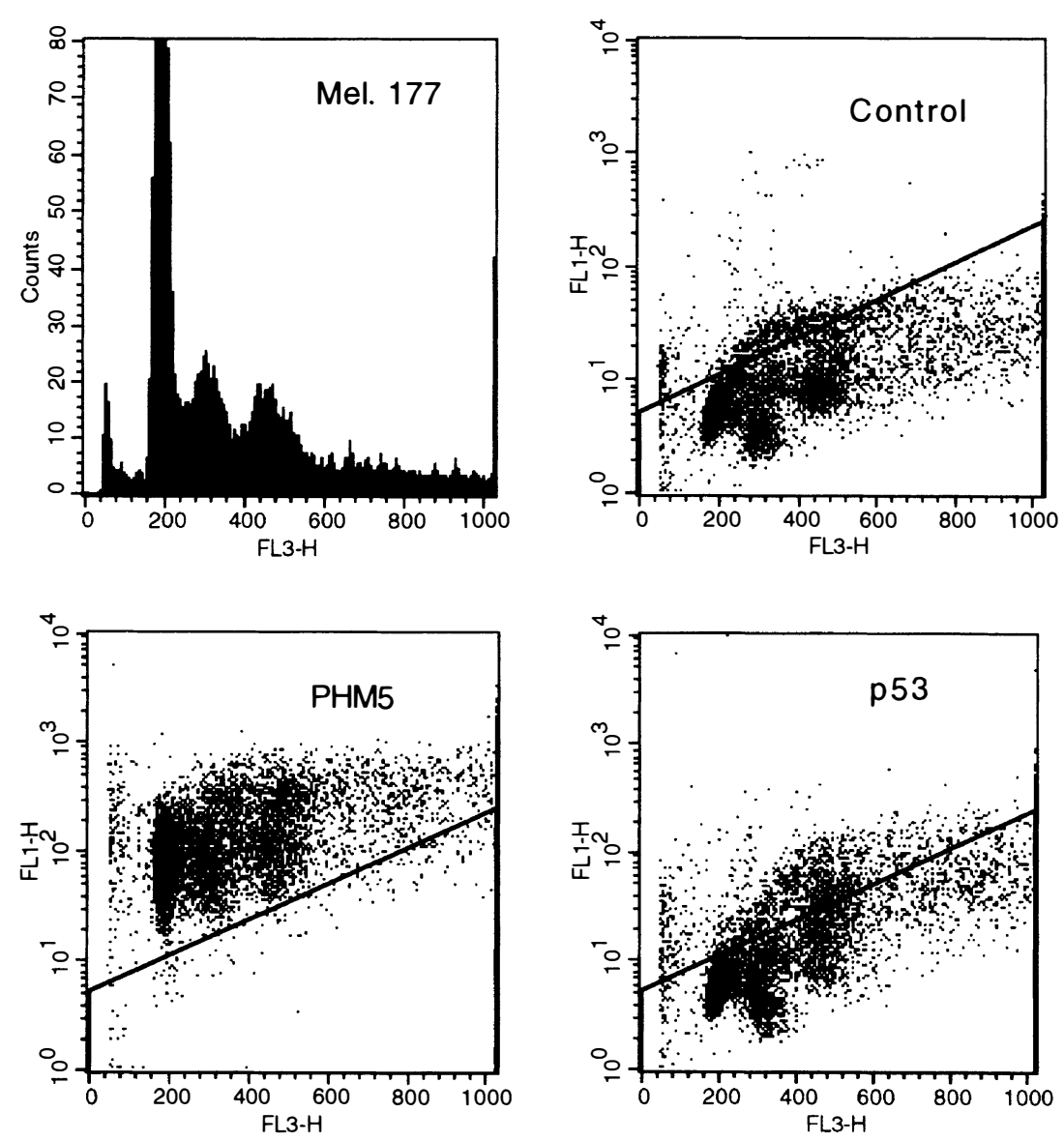

Fig. 3. Mel. 177, an amelanotic choroidal malignant melanoma containing $75 \%$ epitheloid cells, $25 \%$ spindle B cells. The DNA histogram identifies a diploid population together with two aneuploid populations of $5.4 \%$ (DNA Index 1.6) and 20.8\% (DNA Index 2.4) respectively. Dual-parameter dot displays illustrate the staining seen with the negative and positive controls (Control and PHM-5 respectively), and the oncoprotein p53.

(y-axis), on which three regions have been drawn to surround HLA-DR-negative diploid cells amounting to $14.3 \%$ (region 1 ), a discrete population of near-diploid non-HLA staining cells (3.7\%, DNA Index 1.24, region 2) and the main proliferating diploid population amounting to $79.4 \%$ of cells (region 3 ). Histogram B shows the DNA histogram of this sample, and indicates the difficulty in observing these populations by single-parameter analysis. Histograms C, D and E show cells selected by regions R1, R2 and R3 respectively, to provide DNA histograms for ploidy peak analysis; the same regions have also been used to isolate populations of cells to make individual measurements of HLA-DR antibody binding, the results being shown in histograms $F, G$ and $H$.

Not all uveal melanoma samples provided large numbers of cells, the resulting yield being proportional to the initial sample size, rather than an inability to dissociate the tissue sample, so in certain cases the size of the antibody panel had to be contracted. However, in no instance was there ever any problem in dissociating the tumour sample. The only difficulty encountered was in the quality of the $D_{N A} G_{1}$ peak $C V$ in strongly pigmented tumour preparations. No easy solution was found to improve the results, which were thought to be based on steric hindrance preventing total DNA saturation by the DNA dye, propidium iodide, coupled to possible autofluorescence and abnormal amounts of light scattering caused by the strong pigment.

\section{Discussion}

This study shows that flow cytometry can rapidly and accurately phenotype cells in suspension from ocular melanoma tissue. More importantly, it also enables the detailed analysis of complex mixtures of populations within tumours. For example, by distinguishing diploid from aneuploid cells, or cells in each phase of the cell cycle, antibody staining can be related to precise subpopulations of cells within the tumour. Given appropriate markers (antibodies), this approach may enable the identification of sub-populations having a metastatic phenotype, i.e. capable of migration, evasion of the host's immune response and the potential to respond to endogenous growth factors. This technique should therefore be capable of producing individual 'tumour profiles', which may be of greater value for the prediction of patient survival and response to therapy than DNA ploidy and cell cycle measurements alonealthough these parameters are still thought to offer important qualitative and quantitative information on tumour cell proliferation. ${ }^{7}$ 

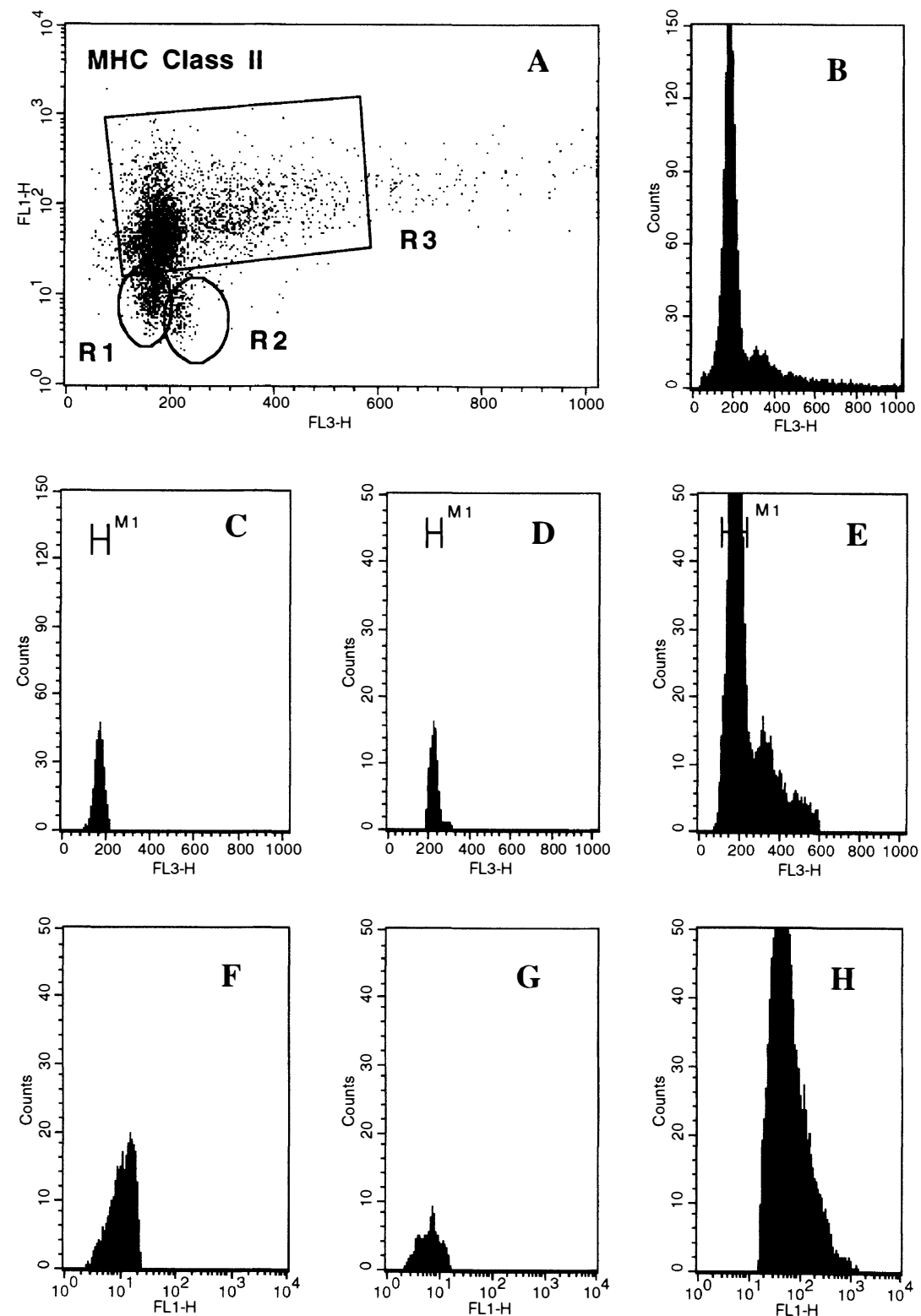

Fig. 4. The value of flow cytometry in enabling the selective analysis of gated populations of cells. The choroidal malignant melanoma illustrated (Mel. 181) has been stained with the MHC Class II antibody, HLA-DR, and the initial data are shown in (A) as a dot display of DNA content ( $\mathrm{x}$ axis) versus HLA-DR staining (y-axis). Three regions have been drawn to surround HLA-DR-negative diploid cells, amounting to $14.3 \%$ (R1); a discretepopulation of near-diploid non-HLA staining cells (3.7\%, DNA Index 1.24, R2); and the main proliferating diploid population, amounting to $79.4 \%$ of cells (R3). (B) The DNA histogram of this sample, indicating the difficulty in observing these populations by single-parameter analysis. (C), (D) and (E) Cells selected by regions R1, R2 and R3 respectively, to provide DNA histograms for ploidy peak analysis; the same regions have also been used to isolate populations of cells to make individual measurements of HLA-DR antibody binding, the results being shown in $(F),(G)$ and $(H)$.

Our results illustrate clear antibody binding for MHC antigens, the adhesion molecule ICAM-1, and the oncoproteins (c-erbB-2, c-myc and bcl-2), which suggests that our protocol is effective for the detection of membrane, cytoplasmic and nuclear antigens. The combination of a fixative and permeabilising agent clearly does not damage or mask antigen to any significant degree, nor do they prevent DNA fluorochrome penetration into the nucleus as it was still possible to measure high-quality DNA profiles using simple DNA staining procedures, from which it was possible to calculate the percentage of cells in each phase of the cell cycle. Populations of cells with differing DNA ploidy values could also be resolved, although this was dependent upon their frequency in the total sample. It was not considered that the resolution of ploidy subgroups was any easier in fresh rather than fixed samples, but the combination of DNA fluorochrome and antibody revealed sub-populations of cells with abnormal DNA content that would otherwise be invisible using singleparameter analysis.

With the range of antibodies available commercially, it is clear that some consideration has to be given to the composition of any antibody panel used to generate a 'tumour profile'. For this reason the pilot study presented here selected a range of potentially useful markers as identified from the literature. In common with the study of Carrel et al., ${ }^{12} \mathrm{MHC}$ Class I and II 
expression was targeted and when antibody binding was detected, usually involved smaller sub-populations of tumour cells within the sample. They reported Class I expression on the majority of spindle and mixed type lesions, and $50 \%$ of the epitheloid type lesions, with generally higher levels of Class II expression than Class I. This was contradicted by Jager et al. ${ }^{13}$ who detected higher levels of HLA Class I antibody (B9-12-1) staining $(75 \%)$ on the ocular melanomas tested, with $44 \%$ of lesions staining for HLA Class II (DR, D1-12). In a further immunohistochemical study, Waard-Siebinga et al. ${ }^{14}$ found all uveal melanomas to have high level HLA Class I (monomorphic determinants), variable expression of polymorphic molecules (A2, A3, BW4, BW6), and low level expression of $\beta 2$-microglobulin and HLA-B locus that was associated with a large tumour diameter. Class II expression was generally low (0-35\%), yet the simple presence of Class II-positive cells has been associated with those melanomas having an increased risk of metastases. ${ }^{15}$ Class II expression has been reported on $50 \%$ of spindle and epitheloid type lesions and low numbers of mixed cell lesions. Natali et al. ${ }^{16}$ found that only mixed and epithelial type lesions expressed HLA Class I, not spindle lesions; and HLA Class II was seen on only one epetheloid and one mixed cell type. The implication of measuring MHC Class I and Class II molecules is clear if patients are to be given immunotherapy, as the presence of MHC Class I on the tumour cells would be a prerequisite for the generation of an immune response by tumour-specific T cells. ${ }^{17}$ Dual-parameter flow cytometry would therefore be a rapid means of quantifying $\mathrm{MHC}$ molecule expression on tumour cell populations.

Adhesion molecules may be responsible for tumour cell escape from the primary site into the circulation, by binding to endothelial cells, enabling contact with and passage through the basement membrane. ${ }^{18}$ Tumour cell/endothelial cell binding may also cause the increased secretion of metalloproteinases, which are enzymes capable of degrading the basement membrane. ${ }^{19,20}$ Intercellular adhesion molecule-I (ICAM-1, CD54) is one such molecule known to be expressed on a range of tumour cells as well as tissue fibroblasts. ICAM-1 may also offer some degree of protection to a tumour cell in the circulation, if adhesion to other cells provides a means of evading the normal host immune response to a foreign cell. The presence of this molecule may therefore be associated with increased survival of tumour cells in the circulation, and ultimately an increased chance for the development of metastases, ${ }^{21}$ as higher levels of ICAM-1 have been reported in metastatic melanoma compared with the primary lesion. $^{22}$

The inhibition of apoptosis by the $b c l-2$ protein may enable tumours to survive longer, develop extensive genotype transformations, and possibly be more aggressive. The $b c l-2$ oncoprotein is encoded by a gene involved in the $14 ; 18$ chromosome translocation that brings the $b c l-2$ gene into juxtaposition with the immunoglobulin heavy chain gene and causes the over- expression of the $b c l-2$ gene..$^{23}$ The protein is reported to be an inner membrane protein, localised within mitochondria. ${ }^{24}$ Our results demonstrate that antibody binding to $b c l-2$ is indeed possible using this technique, and a more detailed study may identify the significance of $b c l-2$ gene expression in uveal melanoma progression, and whether the ability of a cell to prevent the initiation of apoptotic pathways is detrimental to patient survival. The presence of discrete sub-populations of cells having different levels of expression of this protein may be related to an incomplete response to chemo- or radiotherapy with the survival of resistant clones.

The results of the present study may suggest that uveal tumours develop from cells of differing origins, leading to complex variations in the expression of surface molecules, and also their responsiveness to and synthesis of, growth factors and oncoproteins involved in cell cycle control. The protocol presented in this paper offers a rapid, simple and convenient means of enumerating multiple parameters on single samples and, with the careful selection of antibodies, may enable the identification of non-responsive tumours with a poor prognosis at a level previously impossible using standard histological methods.

\section{References}

1. Egan KM, Seddon JM, Glynn RJ, Gragoudas ES, Albert DM Epidemiologic aspects of uveal melanoma. Surv Ophthalmoi 1988;32:239-57.

2. Char DH, Huhta K, Waldman F. DNA cell cycle studies in uveal melanoma. Am J Ophthalmol 1989;107:65-72.

3. Rennier IG, Rees RC, Parsons MA, Lawry J, Cottam D. Estimation of DNA content in uveal melanoma by flow cytometry. Eye 1989;3:611-7.

4. Richardson RPS, Lawry J, Rees RC, Rennie IG. DNA index and \% proliferation in posterior uveal melanoma: a 5-year prospective study of fresh tissue using flow cytometry. Eye 1997;11:629-34.

5. Muhonen T, Pyrhonen S, Laasonen A, Asko-Seljavaara S, Franssila K. DNA aneuploidy and low S-phase fraction as favourable prognostic signs in metastatic melanoma. $\mathrm{Br} \mathrm{J}$ Cancer 1991;64:749-52.

6. Bartkowiak D, Otto F, Schumann J, Lippold A, Drepper H. Sequential DNA flow cytometry in metastatic malignant melanoma. Oncology 1991;48:154-7.

7. Mooy C, Vissers K, Luyten G, Mulder A, Stijnen T, de Jong P, Bosman F. DNA flow cytometry in uveal melanomas: the effect of pre-enucleation irradiation. Br J Ophthalmol 1995;79:174-7.

8. Lawry J, Rogers K, Duncan JL, Potter CW. The identification of informative parameters in the flow cytometric analysis of breast carcinoma. Eur J Cancer 1993;29:719-23.

9. Nowak R, Oelschlaegel U, Schuler U, Zengler H, Hofmann R, Ehninger G, Andreeff M. Sensitivity of combined DNA/ immunophenotype flow cytometry for the detection of low levels of aneuploid lymphoblastic leukaemia cells in bone marrow. Cytometry (Commun Clin Cytometry) 1997;30:47-53

10. Feitz WFJ, Karthaus HFM, Beck HLM, Romun C, Van Der Meyden APM, Debruyne FMJ, et al. Tissue-specific markers in flow cytometry of urological cancers. II. Cytokeratin and vimentin in renal-cell tumours. Int J Cancer 1986;37:201-7. 
11. Park $\mathrm{CH}$, Kimler BF. Tumour cell-selective flow cytometric analysis for DNA content and cytokeratin expression of clinical tumour specimens by 'cross-gating'. Anti-cancer Res 1994;14:29-36.

12. Carrel S, Schreyer M, Gross N, Zografos L. Surface antigenic profile of uveal melanoma lesions analysed with a panel of monoclonal antibodies directed against cutaneous melanoma. Anti-cancer Res 1990;10:81-90.

13. Jager MJ, Van der Pol JP, de Wolff-Rouendaal D, De Jong PTVM, Ruiter DJ. Decreased expression of HLA Class II antigens on human uveal melanoma cells after in vivo X-ray irradiation. Am J Ophthalmol 1988;105:78-86.

14. Waard-Siebinga I, Houbiers JGA, Hilders CGJM, WolffRouendaal D, Jager MJ. Differential expression of HLA-A and B-alleles on uveal melanoma as determined by immunohistology. Ocular Immunol Inflammation 1996;4:1-14.

15. Zaloudik J, Moore M, Ghosh AK, Mechl Z, Rejthar A. DNA content and MHC class II antigen expression in malignant melanoma: clinical course. J Clin Pathol 1988;41:1078-84.

16. Natali PG, Bigotti A, Nicotra MR, Nardi RM, Delovu A, Segatto $\mathrm{O}$, Ferrone $\mathrm{S}$. Analysis of the antigenic profile of uveal melanoma lesions with anti-cutaneous melanomaassociated antigen and HLA monoclonal antibodies. Cancer Res 1989;49:1269-74.

17. Tran JM, Murray TG, Uno T, Valore N, Ksander BR. Expression of HLA Class I on ocular melanoma during progression from primary to metastatic disease. Invest Ophthalmol Vis Sci 1996;37:s208.
18. Juliano RL. The role of $\beta_{1}$ integrins in tumours. Semin Cancer Biol 1993;4:277-83.

19. Cottam DW, Rennie IG, Woods K, Parsons A, Bunning RAD, Rees RC. Gelatinolytic metalloproteinase secretion patterns occur in ocular melanoma. Invest Ophthalmol Vis Sci 1992;33:1923-7.

20. Cottam DW, Rees RC. Regulation of matrix metalloproteinases: their role in tumour invasion and metastases. Int J Oncol 1993;2:861-72.

21. Johnson JP, Stade BG, Holzmann B, Schwable W, Riethmuller G. De novo expression of intercellular-adhesion molecule 1 in melanoma correlates with increased risk of metastases. Proc Natl Acad Sci USA 1989;86:641-4.

22. Natali P, Nicotra MR, Cavaliere R, Bigotti A, Romano G, Temponi M, Ferrone S. Differential expression of intercellular adhesion molecule 1 in primary and metastatic melanoma lesions. Cancer Res 1990;50:1271-8.

23. Pezzella F, Tse AGD, Cordell JL, Pulford KAF, Gatter KC, Mason DY. Expression of the $b c l-2$ oncoprotein is not specific for the 14;18 chromosome translocation. Am J Pathol 1990;137:225-32.

24. Hockenbery D, Nunez G, Milliman C, Schreiber RD, Korsmeyer SJ. $b c l-2$ is an inner mitochondrial membrane protein that blocks programmed cell death. Nature 1990;348:334-6. 